Sir,

\section{Response to Jones et al}

We note with interest the paper by Jones et al regarding the role of General Practitioners with specialist interests (GPwSI) in reducing the high false-positive rate of referrals for glaucoma. ${ }^{1}$ They maintain that the reduced false-positive rate in their study translates into improved efficiency. However, efficiency is characterised by maximum effectiveness with minimum waste of resources. Measurement of efficiency would also require demonstration of a reduced false-negative rate. It would be of interest to know how many of the patients discharged by the GPwSI would also have been discharged by the glaucoma specialist.

\section{Reference}

1 Jones LD, Robinson R, Cook NJ. General practitioner with a special interest improves the efficiency of glaucoma referrals. Eye 2006; 20: 942.

NJ Crosby and T Gillow

Department of Ophthalmology,

University Hospitals of North Staffordshire,

Stoke-on-Trent, Staffordshire, UK

Correspondence: NJ Crosby,

Department of Ophthalmology,

University Hospitals of North Staffordshire,

Princes Road, Stoke-on-Trent,

Staffordshire ST4 7LS, UK

Tel: +44 1782 555153;

Fax: + 441782554297 .

E-mail: niall_crosby@hotmail.com

Proprietary interests or research funding: None.

Eye (2007) 21, 676. doi:10.1038/sj.eye.6702682;

published online 9 February 2007

Sir,

\section{Sildenafil-associated vascular CASUALTIES}

In a recent issue of the Eye, I read with interest the correspondence regarding sildenafil (Viagra) and nonarteritic anterior ischaemic optic neuropathy (NAION), cilioretinal artery occlusion and central retinal vein occlusion in a haemodialysis patient. Dr Gedik et $\mathrm{al}^{1}$ discuss the causal relationship between drug and its results. I think their correspondence guides the scientists in the adverse effects of drugs. On the other hand, phosphodiesterase (PDE)-5 inhibitors, currently used as on-demand drugs, could be administered as prophylaxis agents either for erectile dysfunction, which is mainly a neurovascular disease, or for some other widespread vascular diseases owing to their beneficial effects on endothelial functions. ${ }^{2}$ By the way, I believe it merits mention to share the results of our sildenafil studies with readers. ${ }^{3-5}$ In the first study, we performed the cotton thread, Schirmer I tests, and tear break-up time to determine the acute effects of sildenafil on tear functions. The tests were applied to the subjects just before and $1 \mathrm{~h}$ after ingesting oral sildenafil $50 \mathrm{mg}$. The results of these tests were insignificant in comparison with the values of pre- and postmedication of sildenafil. ${ }^{3}$ The evaluation of the acute effects on tear functions at the peak drug serum levels was important because erectile dysfunction and abnormal tear functions are generally seen in an older population. Also, we evaluated the long-term effects of the same drug on visual acuity, colour vision, intraocular pressure, electroretinography (amplitude and implicit time of b-wave), blue-on-yellow and white-on-white Humphrey visual field, and tear functions in chronic users who had received one or more times in a week for more than 3 months at least, but not in the last $12 \mathrm{~h}$ before the study. We had not detected any abnormalities on the above-mentioned tests in sildenafil users and concluded that repeated exposures of ocular tissues to therapeutic doses of sildenafil were unlikely to impair their functions. ${ }^{4}$

Overall, PDE-5 inhibitors have ameliorating effects on endothelial functions therefore, the cause of NAION can not be explained by detrimental effects on the vascular supply of the optic nerve. It is not possible to say whether sildenafil is directly responsible in these events, as other factors could also have been involved in NAION, cilioretinal artery occlusion, and central retinal vein occlusion. More clinical/experimental multidisciplinary studies are needed to clarify these issues. I think the patients using sildenafil should closely collaborate with the ophthalmologist and appeal to them when any visual symptom is experienced.

\section{References}

1 Gedik S, Yilmaz G, Akova YA. Sildenafil-associated consecutive nonarteritic anterior ischaemic optic neuropathy, cilioretinal artery occlusion, and central retinal vein occlusion in a haemodialysis patient. Eye 2006 May 26.

2 Frajese GV, Pozzi F. New achievement and novel therapeutic applications of PDE5 inhibitors in older males. J Endocrinol Invest 2005; 28: 45-50.

3 Oguz H, Verit A, Gurkan T, Yeni E. The acute effects of sildenafil on tear functions. Ann Ophthalmol 2005; 37: 281-284. 